\title{
INFLUÊNCIA DA DISTÂNCIA ENTRE PASSADAS DE COLHEDORA EQUIPADA COM MONITOR DE COLHEITA NA PRECISÃO DOS MAPAS DE PRODUTIVIDADE NA CULTURA DO MILHO
}

\author{
CLÁUdIO L. BAZZI ${ }^{1}$, EDUARDO G. SOUZA², MIGUEL A. URIBE OPAZO ${ }^{3}$, \\ LÚCIA H. P. NÓBREGA ${ }^{4}$, RAIMUNDO PINHEIRO NETO ${ }^{5}$
}

\begin{abstract}
RESUMO: Um dos entraves para a maior expansão da agricultura de precisão está, principalmente, no elevado custo dos equipamentos a serem utilizados. A geração de mapas de produtividade é a fase de mais fácil execução na agricultura de precisão. Entretanto, quando várias colhedoras operam simultaneamente em uma determinada área, nem sempre é possível dispor de monitores de colheita em todas as máquinas. Assim, neste trabalho, foi avaliado o decréscimo da precisão em mapas de produtividade obtidos a partir de áreas onde se utilizaram colhedoras não-equipadas com monitor de colheita associadas a colhedoras monitoradas. Também foram comparados os métodos de interpolação "inverso da distância ao quadrado" e "krigagem". Inicialmente, a interpolação do mapa com todos os pontos de amostragem (original) foi gerada e, a partir dessa, foi feita a eliminação de passadas de modo a obter os mapas simulados. Na comparação entre os mapas simulados e o original, foi utilizado o coeficiente de desvio relativo - CDR, e o índice kappa. O número de passadas eliminadas diminuiu a precisão dos mapas em ambos os métodos de interpolação. Sendo assim, a quantidade mínima necessária de monitores em um conjunto de colhedoras depende da precisão desejada. Portanto, as máquinas devem ser intercaladas de maneira que as amostras sejam coletadas uniformemente por toda a área a ser colhida.
\end{abstract}

PALAVRAS-CHAVE: agricultura de precisão, métodos de interpolação, mapas temáticos.

\section{INFLUENCE OF DISTANCE BETWEEN COMBINES EQUIPPED WITH YIELD MONITORS ON THE PRECISION OF YIELD MAPS FOR CORN CROPS}

\begin{abstract}
One of the main obstacles to the larger expansion of precision agriculture is the high cost of the equipments to be used. The generation of yield maps is the easiest step in the sequence of the precision agriculture process. Nevertheless, the availability of yield monitors for all the machines is not always possible when several combines are concomitantly harvesting in a given area. Within this context, this research was carried out aiming to evaluate the decrease of precision of yield maps obtained from data collected in areas where combines not equipped with yield monitors were harvesting concomitantly with combines equipped with such device. Two different interpolation methods, the "inverse of the square distance" and the "kriging", were also evaluated. Initially, the interpolation of the map with all sampling sites (original) was generated, and from that, the elimination of combine steps was performed, therefore originating the simulated maps. To compare the original maps with the simulated ones, the coefficient of relative deviation and the Kappa index were used. The number of eliminated combine steps decreased the precision of yield maps as demonstrated by both interpolation used methods. Therefore, the minimum number of monitors needed in a group of combines depends on the desired precision. Thus, the intercalation of monitored and non-monitored combines should be performed so that the samples are uniformly collected within all the area to be harvested.
\end{abstract}

KEYWORDS: precision agriculture, interpolation methods, thematic maps.

\footnotetext{
${ }^{1}$ Tecnólogo em Processamento de Dados, M.Sc., Programa de Pós-Graduação em Engenharia Agrícola, CCET - UNIOESTE/ Cascavel - PR, claudiobazzi@yahoo.com.br

${ }^{2}$ Eng $^{\circ}$ Mecânico, Prof. Associado, CCET/UNIOESTE, Grupo de Pesquisa GROSAP, Cascavel - PR.

${ }^{3}$ Estatístico, Professor Associado, CCET/UNIOESTE, Grupo de Pesquisa GGEA, Cascavel - PR.

${ }^{4}$ Eng $^{\mathrm{a}}$ Agrônoma, Prof. Adjunta, CCET/UNIOESTE, Grupo de Pesquisa GROSAP, Cascavel - PR.

${ }^{5}$ Eng ${ }^{\circ}$ Agrícola, Prof. Adjunto do Departamento de Agronomia da UEM, Maringá, PR
}

Recebido pelo Conselho Editorial em: 27-4-2007

Aprovado pelo Conselho Editorial em: 10-3-2008 


\section{INTRODUÇÃO}

A agricultura de precisão (AP), segundo VETTORAZZI \& FERRAZ (2000), baseia-se na coleta e na análise de dados geoespaciais, viabilizando intervenções localizadas no cultivo, com a exatidão e a precisão adequadas. As técnicas de geoprocessamento subsidiam a identificação e a correlação das variáveis que afetam a produtividade, por meio da sobreposição, do cruzamento e da regressão, em sistemas de informação geográfica (SIGs), de mapas digitais do relevo, dos atributos do solo e da produtividade.

BALASTREIRE (1998) considerou que a geração de mapas de produtividade é a fase que apresenta maior facilidade de execução dentro do ciclo da AP. Devido à grande variedade de equipamentos e pesquisas realizadas, principalmente em colheita de cereais, tais como, soja e milho, pode-se coletar grande quantidade de informações sobre uma área a um custo operacional acessível (SCHUELLER, 2000).

SHIRATSUCHI et al. (2004) avaliaram o efeito do espaçamento entre faixas de colheita e sua conseqüência na geração de mapas de produtividade de milho e concluíram que se pode pular de quatro a seis faixas de 5,6 m, e que o método de interpolação krigagem é mais robusto ao aumento do número de passadas. TAYLOR et al. (2000) não encontraram diferença ao comparar tamanho de grades (grid) de 2 a 4 vezes a largura da plataforma e que uma grade de 2 vezes a largura da plataforma não compromete o detalhe de informação do mapa de produtividade e diminui a quantidade de dados coletados e armazenados eletronicamente.

Nesse contexto, este trabalho avaliou o decréscimo da precisão nos mapas de produtividade pela utilização, na mesma área, simultaneamente, de colhedoras não-equipadas com monitor de colheita associadas a colhedoras monitoradas. Compararam-se, também, os métodos de interpolação "inverso da distância ao quadrado" e "krigagem".

\section{MATERIAL E MÉTODOS}

Os dados de produtividade utilizados nesta pesquisa foram coletados em propriedade rural no município de Cascavel, Estado do Paraná, com localização geográfica $24^{\circ} 58^{\prime} 44,4^{\prime \prime} \mathrm{S}$ e $53^{\circ} 31^{\prime} 26,4^{\prime \prime} \mathrm{W}$, e altitude média de $650 \mathrm{~m}$. A cultura comercial utilizada foi o milho, com ciclo fisiológico de aproximadamente 120 dias, semeada entre os dias 25 e 30 de janeiro de 2004, em plantio direto, com espaçamento entre plantas de $0,20 \mathrm{~m}$ e entre linhas de $0,70 \mathrm{~m}$.

A colheita foi realizada de 30 de junho a 2 de julho de 2004, em área de 32,8 ha, de onde foi selecionada uma subárea de 13,2 ha para ser analisada neste trabalho. A densidade de pontos coletados foi de 1.113 pontos por ha, obtida pela utilização do intervalo de leitura do monitor de colheita de 3 segundos e velocidade média de deslocamento da colhedora de $5 \mathrm{~km} \mathrm{~h}^{-1}$. O total de pontos coletados, sem qualquer remoção de erros, correspondeu a 14.693. O monitor de colheita utilizado foi o AgLeader ${ }^{\circledR}$, modelo PF $3000^{\circledR}$, montado no ano de 2001 em colhedora New Holland TC $57^{\circledR}$, ano de fabricação 1994, com potência nominal de $124 \mathrm{~kW}$, equipada com plataforma despigadora de milho, com seis linhas espaçadas entre si de $0,70 \mathrm{~m}$ e capacidade de armazenamento no graneleiro de $5.000 \mathrm{~L}$.

Após a coleta dos dados pelo monitor de colheita, os mesmos foram gravados em cartão PCMCIA, disponível no próprio monitor, e exportados para um computador tipo PC (Personal Computer) para a realização das filtragens, geração e visualização dos mapas de produtividade. Na filtragem dos dados, foram eliminados pontos amostrais que apresentavam produtividade mais elevada (acima $8.076 \mathrm{~kg} \mathrm{ha}^{-1}$ ) ou mais baixa (abaixo de $1.969 \mathrm{~kg} \mathrm{ha}^{-1}$ ), encontradas por meio de análise estatística exploratória e que, provavelmente, foram influenciados por fontes de erros, tais como tempo de atraso, tempo de enchimento e esvaziamento, posicionamento do GPS e largura efetiva de colheita menor que a apresentada ao monitor (MICHELAN, 2005).

Concluída a eliminação de dados inconsistentes, verificou-se que, do total de 14.693 pontos coletados no processo de colheita, restaram 13.473 pontos (1.220 pontos foram eliminados), dando 
origem ao mapa de pontos, definido como "mapa original" (Figura 1). O mapa de pontos apresenta somente a produtividade em cada ponto. A partir desse, podem-se confeccionar os mapas realizando a interpolação dos pontos, dando origem aos mapas de contorno, que permitem melhor análise (Figura 2).

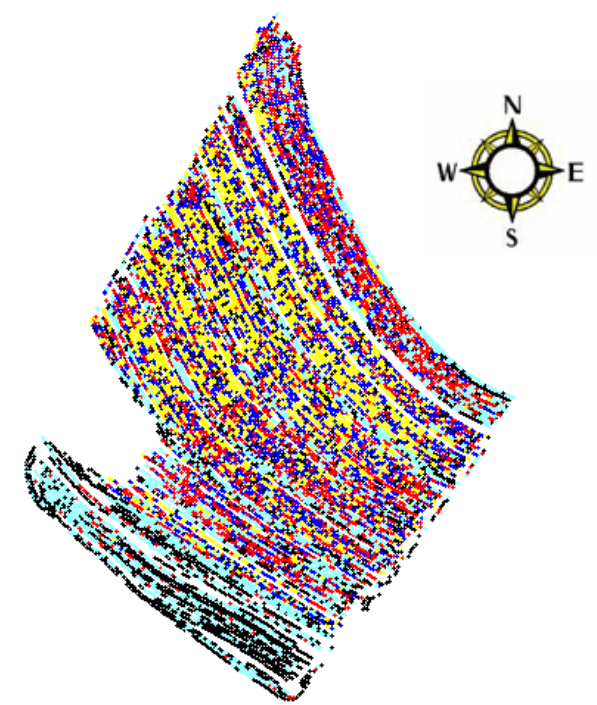

FIGURA 1. Mapa de produtividade $\left(\mathrm{kg} \mathrm{ha}^{-1}\right)$ : mapa de pontos, definido como "mapa original", com 13.473 pontos de coleta de dados, com valores não-interpolados. Yield map $\left(\mathrm{kg} \mathrm{ha}^{-1}\right)$ : point map defined as the "original map" with 13,473 data points, with noninterpolated values.

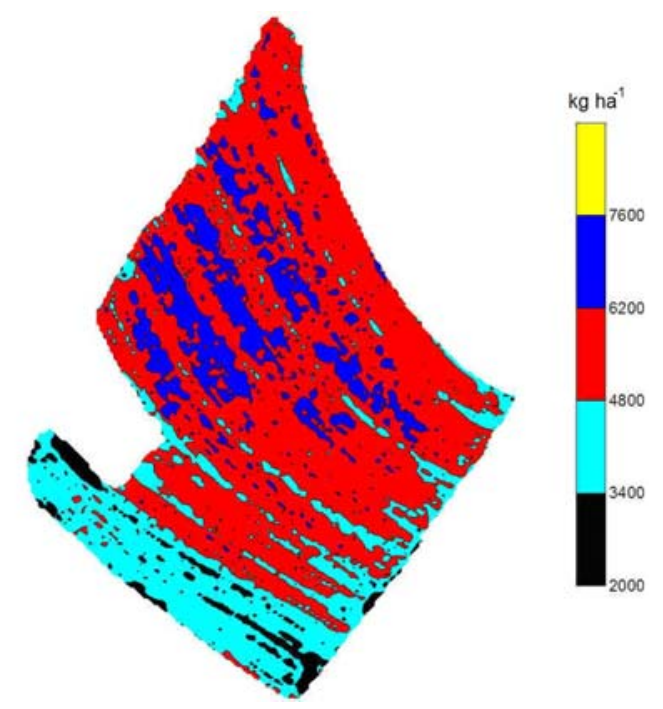

FIGURA 2. Mapa de produtividade $\left(\mathrm{kg} \mathrm{ha}{ }^{-1}\right)$ : mapa de contorno, definido como "mapa original", interpolado pelo método do inverso da distância ao quadrado. Yield map $\left(\mathrm{kg} \mathrm{ha}^{-1}\right)$ : contour map defined as "the original map" and interpolated by the kriging method.

Depois da filtragem dos dados, foram gerados conjuntos de dados simulando comboios de colhedoras, sendo que algumas delas possuíam monitor de colheita, e outras não. No conjunto original, todas as colhedoras possuíam monitor. No conjunto $1: 1$, havia uma colhedora monitorada. No conjunto $2: 1$, duas colhedoras no total, mas somente uma possuía monitor, e assim por diante (Tabela 1 e Figuras 3 e 4).

Os dados de cada conjunto foram analisados estatisticamente por meio de análise exploratória da estatística descritiva, calculando-se a média, a mediana, o quartis, o mínimo, o máximo, o desvio-padrão e o coeficiente de variação. Para a verificação da normalidade dos dados, aplicaramse os testes de normalidade de Anderson-Darling e de Kolmogorov-Smirnovs. Foram considerados com distribuição de probabilidade normal os dados que apresentaram resultado de p-valor maior que 0,05 , ou seja, a $5 \%$ de significância em, pelo menos, um dos testes.

$\mathrm{Na}$ análise geoestatística, construíram-se os semivariogramas para verificar as influências de dependência espacial entre as amostras. Para estimar as estruturas da função semivariância experimental, utilizou-se do estimador proposto por CRESSIE \& HAWKINS (1980). Os semivariogramas experimentais foram obtidos aplicando-se os métodos de ajuste de mínimos quadrados ordinários (OLS) e adotando-se o modelo isotrópico (semivariograma omnidirecional) com um cutoff de 50\% da área máxima. Utilizou-se, nessa análise, do software VESPER 1.6 Demo, fornecido pelo Centro Australiano de Agricultura de Precisão desde 2002. 
TABELA 1. Definição dos tratamentos a serem estudados após eliminação das passadas de colheita. Definition of the treatments to be studied after elimination of combine steps.

\begin{tabular}{cccc}
\hline Conjunto de dados & $\begin{array}{c}\text { Passadas de colheita } \\
\text { excluídas }\end{array}$ & $\begin{array}{c}\text { \% Restante do } \\
\text { número de pontos }\end{array}$ & $\begin{array}{c}\text { Relação entre o total de } \\
\text { colhedoras e colhedoras } \\
\text { monitoradas }\end{array}$ \\
\hline Original & 0 & $100 \%$ & $1: 1$ \\
1 passada & 1 & $50 \%$ & $2: 1$ \\
2 passadas & 2 & $33 \%$ & $3: 1$ \\
3 passadas & 3 & $25 \%$ & $4: 1$ \\
4 passadas & 4 & $20 \%$ & $5: 1$ \\
5 passadas & 5 & $16 \%$ & $6: 1$ \\
6 passadas & 6 & $14 \%$ & $7: 1$ \\
7 passadas & 7 & $12 \%$ & $8: 1$ \\
10 passadas & 10 & $9 \%$ & $11: 1$ \\
15 passadas & 15 & $6 \%$ & $16: 1$ \\
20 passadas & 20 & $4,5 \%$ & $21: 1$ \\
25 passadas & 25 & $4 \%$ & $26: 1$ \\
50 passadas & 50 & $2 \%$ & $51: 1$ \\
100 passadas & 100 & $1 \%$ & $101: 1$ \\
\hline
\end{tabular}

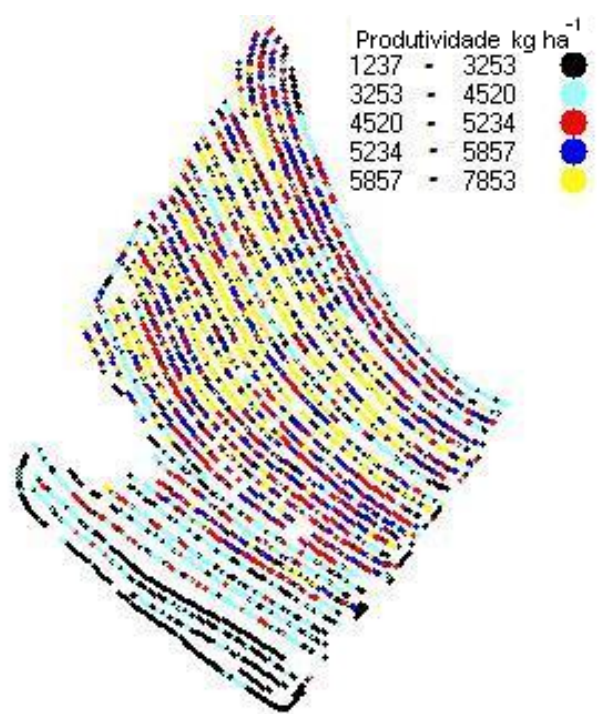

FIGURA 3. Mapa definido como "mapa duas passadas excluídas", com 5.257 pontos de coleta de dados, obtidos pela eliminação de duas passadas da colhedora a cada três passadas. Map defined as "two eliminated step map" with 5,257 data collection points, obtained by the elimination of two combine steps every three steps.

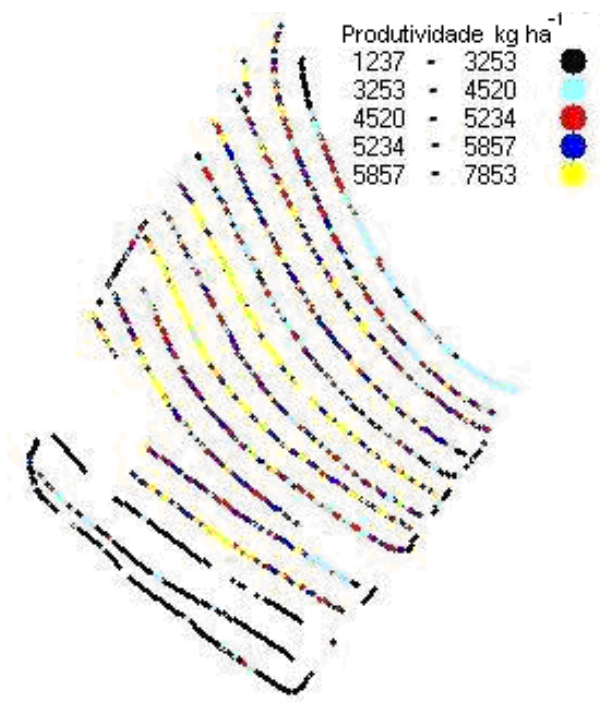

FIGURA 4. Mapa definido como "mapa sete passadas excluídas", com 1.965 pontos de coleta de dados obtidos através da eliminação de sete passadas da colhedora a cada oito passadas. Map defined as "seven eliminated step map" with 1,965 data collection points, obtained by the elimination of seven combine steps every eight steps.

Os mapas temáticos foram construídos para cada conjunto de dados, utilizando-se do software SURFER 8.0 Demo, disponibilizado pela Golden Software. A comparação dos mapas gerados pela eliminação de passadas com o mapa original foi feita utilizando o coeficiente de desvio relativo [CDR, eq.(1)], proposto por COELHO (2006), que expressa a diferença média, em módulo, dos valores interpolados em cada mapa, considerando um deles como o mapa-padrão. Outra técnica de comparação utilizada foi o índice kappa [eq.(2)], proposto por COHEN (1960). Esse método utiliza 
uma matriz de confusão ou matriz de erro, calculando-se, posteriormente, o índice kappa de concordância (CONGALTON, 1991).

$$
\mathrm{CDR}=\sum_{\mathrm{i}=1}^{\mathrm{n}}\left|\frac{\mathrm{P}_{\mathrm{ij}}-\mathrm{P}_{\mathrm{ipad}}}{\mathrm{P}_{\mathrm{ipad}}}\right| \frac{100}{\mathrm{n}}
$$

em que,

$\mathrm{n}$ - número de pontos;

$\mathrm{P}_{\text {ipad }}$ - produtividade no ponto i para o mapa-padrão, e

$\mathrm{P}_{\mathrm{ij}}$ - produtividade no ponto i para o mapa $\mathrm{j}$.

$$
\mathrm{K}=\frac{\mathrm{N} \sum_{\mathrm{i}=1}^{\mathrm{r}} \mathrm{x}_{\mathrm{ii}}-\sum_{\mathrm{i}=1}^{\mathrm{r}}\left(\mathrm{x}_{\mathrm{i}+} \mathrm{x}_{+\mathrm{i}}\right)}{\mathrm{N}^{2}-\sum_{\mathrm{i}-1}^{\mathrm{r}}\left(\mathrm{x}_{\mathrm{i}+}+\mathrm{x}_{+\mathrm{i}}\right)}
$$

em que,

K - índice Kappa de concordância;

$\mathrm{N}$ - número de observações (pontos amostrais);

r- número de linhas da matriz de erro;

$\mathrm{x}_{\mathrm{ii}}$ - observações da linha i e coluna $\mathrm{i}$;

$\mathrm{x}_{\mathrm{i}_{+}}$- total marginal da linha $\mathrm{i}, \mathrm{e}$

$\mathrm{x}_{+\mathrm{i}}$ - total marginal da coluna $\mathrm{i}$.

\section{RESULTADOS E DISCUSSÃO}

Pelo coeficiente de variação (C.V.) obtido por meio da análise estatística descritiva dos conjuntos de dados de produtividade (Tabela 1$)$, com média variabilidade $(20 \% \leq \mathrm{C} . \mathrm{V} . \leq 30 \%)$, verificou-se relativa homogeneidade dos dados (GOMES, 2000). A média da produtividade variou de 5.134 a $5.430 \mathrm{~kg} \mathrm{ha}^{-1}$, diferindo menos que $6 \%$ entre elas. Pelos testes de normalidade realizados, verificou-se que os dados de produtividade, nos conjuntos de dados, não apresentaram distribuição normal de probabilidade, a 5\% de significância. Verificou-se, ainda, média a forte dependência espacial, visto que o coeficiente de efeito pepita variou entre 38,0\% (uma passada) e 6,23\% (20 passadas).

A partir dos parâmetros ajustados aos semivariogramas experimentais, foram criados 28 mapas temáticos para produtividade, sendo 14 pelo método da krigagem (Figura 5) e 14 pelo método do inverso da distância ao quadrado - IQD (Figura 6).

Os coeficientes de desvio relativos (CDR), resultantes da comparação entre os mapas simulados e o original, mostraram aumento gradativo do desvio à medida que aumenta o número de passadas eliminadas, em ambos os métodos de interpolação (inverso da distância ao quadrado e krigagem). Esse aumento do erro foi menor para o método do inverso da distância ao quadrado (Figura 7), evidenciando que o método da krigagem foi mais influenciado pela eliminação dos pontos de amostragem. 

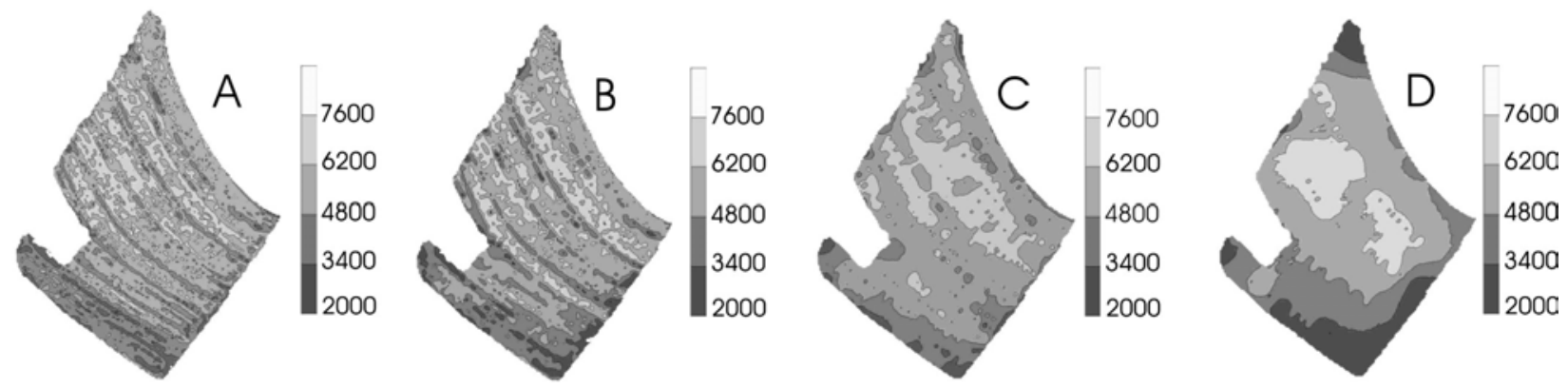

FIGURA 5. Mapas de produtividade $\left(\mathrm{kg} \mathrm{ha}^{-1}\right)$ criados pelo método de interpolação krigagem: a) original; b) "Mapa 1/1 - uma colhedora monitorada e uma não"; c) "Mapa 1/5 - uma colhedora monitorada e cinco não"; d) "Mapa 1/25 - uma colhedora monitorada e 25 não". Yield maps $\left(\mathrm{kg} \mathrm{ha}^{-1}\right)$ created by using kriging: a) original; b) "Map 1/1 - a monitored and a non-monitored combine"; c) "Map 1/5 -a monitored combine and five not"; d) "Map 1/25 - a monitored combine and twenty-five not".
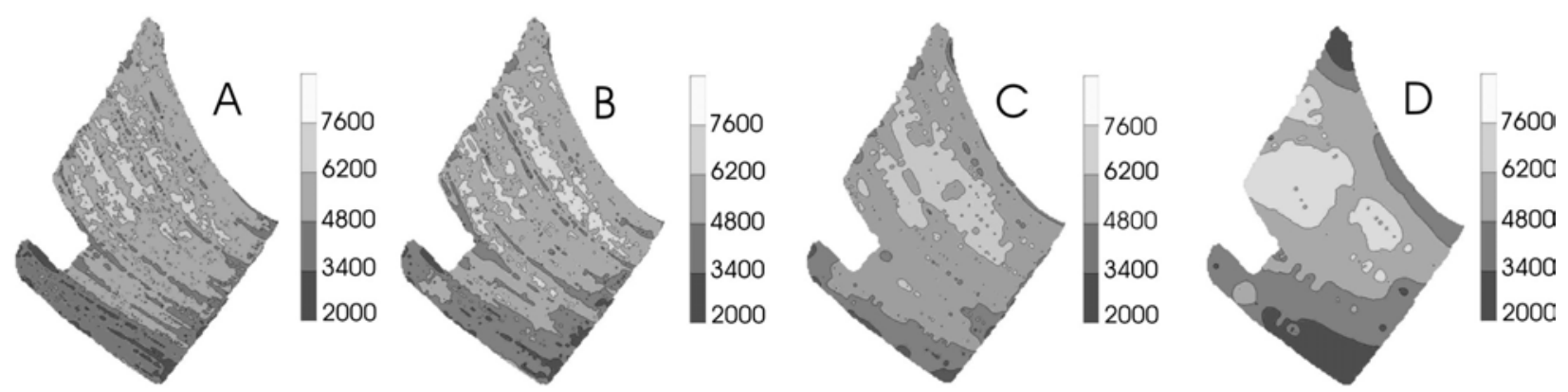

FIGURA 6. Mapas de produtividade $\left(\mathrm{kg} \mathrm{ha}^{-1}\right)$ criados pelo método de interpolação inverso da distância ao quadrado: a) original; b) "Mapa 1/1 - uma colhedora monitorada e uma não"; c) "Mapa 1/5 - uma colhedora monitorada e cinco não"; d) "Mapa 1/25 - uma colhedora monitorada e 25 não". Yield maps $\left(\mathrm{kg} \mathrm{ha}^{-1}\right)$ created by using inverse interpolation method of the square distance: a) original; b) "Map 1/1 - a monitored combine and one not"; c) "Map 1/5 - a monitored combine and five not"; d) "Map 1/25 - a monitored combine and twenty-five not".

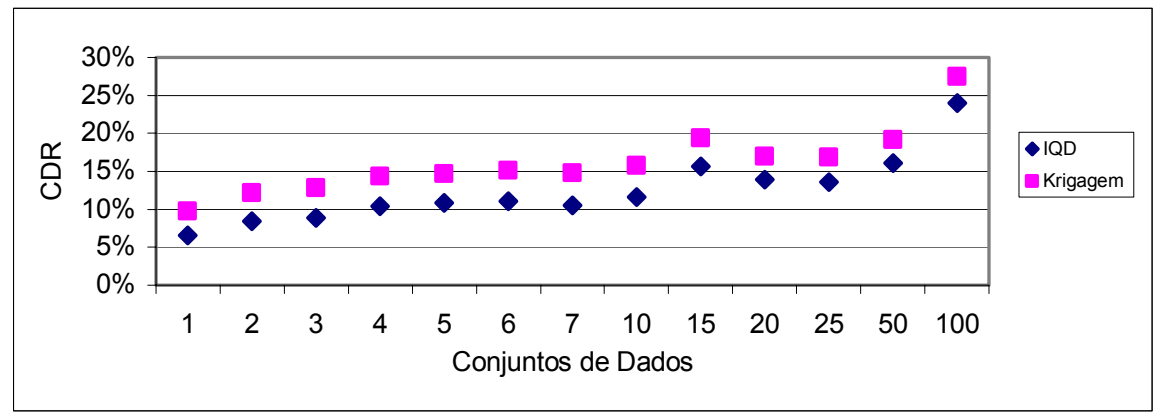

FIGURA 7. Desvio relativo medido pelo método CDR de comparação nas interpolações realizadas pelos métodos do inverso da distância ao quadrado (IQD) e da krigagem. Coefficient of relative deviation (CDR) in function of the interpolation method: inverse of the square distance (IQD) and kriging. 
O desvio relativo entre os mapas de produtividade simulados pelo inverso da distância ao quadrado e por krigagem manteve-se relativamente constante com o aumento do número de passadas retiradas do mapa.

Os índices kappa (Figura 8), resultantes da comparação entre os mapas de produtividade simulados e o original, diminuíram progressivamente à medida que aumentou o número de passadas eliminadas para ambos os métodos de interpolação (inverso da distância ao quadrado e krigagem). O índice kappa foi sempre maior para o método do inverso da distância ao quadrado, significando que o método da krigagem foi mais influenciado pela eliminação dos pontos de amostragem, devido a seu método ser mais robusto. O índice kappa apresentou-se baixo, confirmando a baixa similaridade entre os mapas.

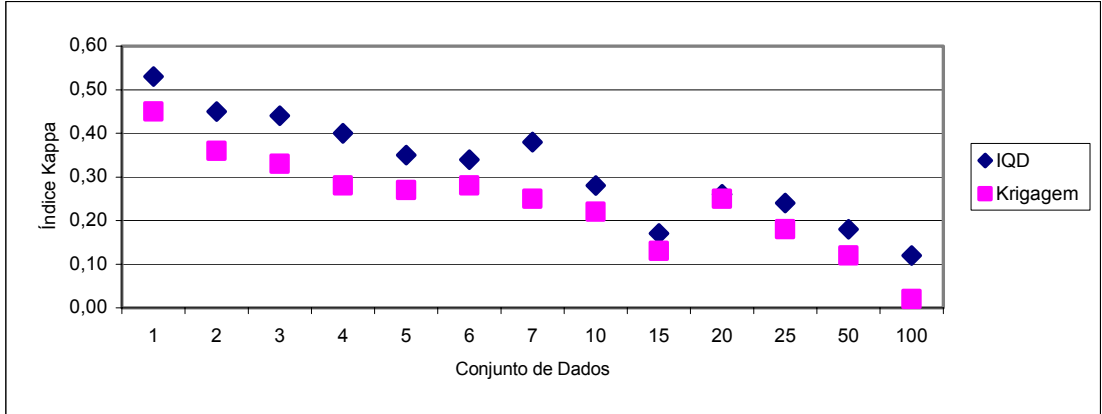

FIGURA 8. Índice kappa de comparação nas interpolações realizadas pelos métodos do inverso da distância ao quadrado (IQD) e da krigagem. Kappa index in function of the interpolation method: inverse of the square distance (IQD) and kriging.

O índice kappa entre os mapas de produtividade simulados pelo inverso da distância ao quadrado e por krigagem (Figura 9) manteve-se relativamente constante com o aumento do número de passadas retiradas do mapa, apresentando valor médio de 0,76 , indicando pequena influência nos métodos de interpolação.

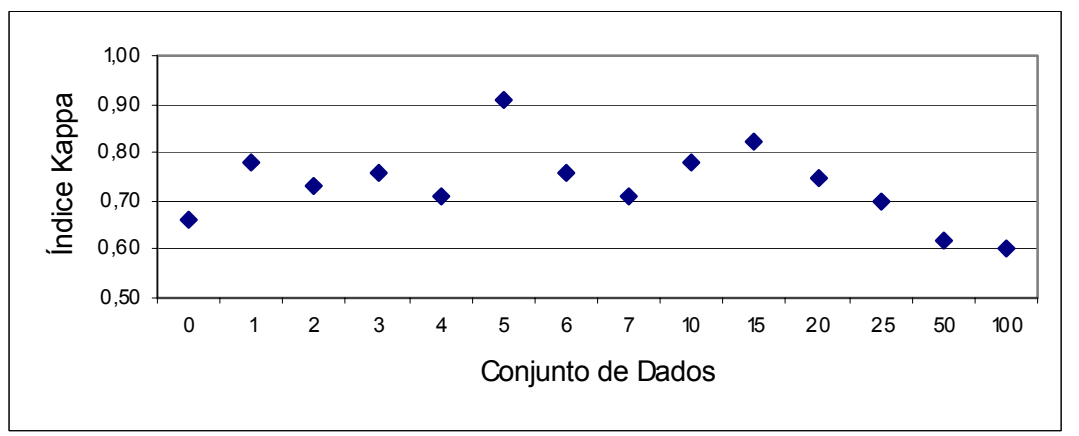

FIGURA 9. Índice kappa entre os mapas de produtividade criados pelos métodos de krigagem e do inverso da distância ao quadrado. Index kappa between yield maps created by kriging and inverse of the square distance methods.

Verifica-se que, nas comparações realizadas, tanto pelo método CDR como pelo kappa, os resultados foram similares (Figuras 10 e 11), visto que o índice kappa apresentou correlação linear com o CDR, com $\mathrm{R}^{2}$ entre 0,940 e 0,966 , ou seja, com explicação do índice kappa acima de $86 \%$. A razão pela qual, apesar de significativa parte dos mapas criados apresentarem índice kappa razoável ou ruim (kappa $<0,40$; LANDIS \& $\mathrm{KOCH}, 1977)$, mas manterem boa correlação com o CDR $\left(\mathrm{R}^{2}>0,94\right)$, é que, apesar de dois mapas estarem bem correlacionados, o percentual de regiões coincidentes pode ser baixo (baixo índice kappa). 


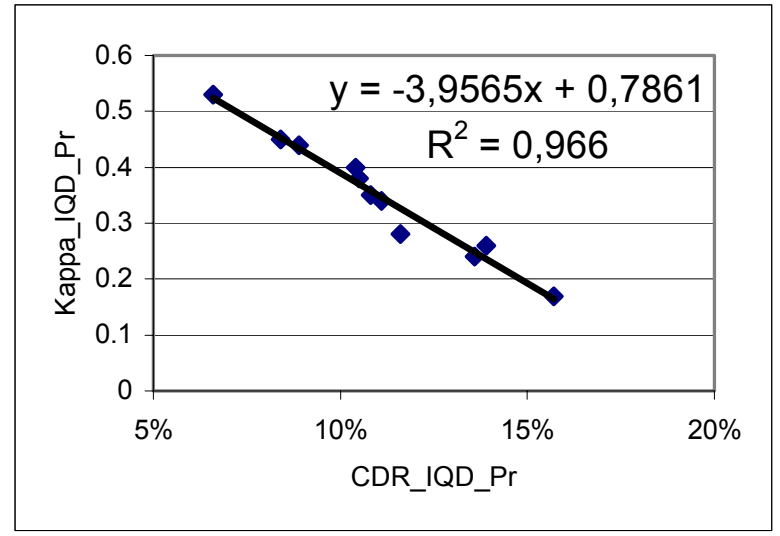

FIGURA 10. Índice kappa para os mapas FIGURA 11. Índice kappa para os mapas interpolados por inverso da distância ao quadrado (kappa_IQD_Pr), em função do coeficiente de desvio relativo para os mapas interpolados por inverso da distância ao quadrado (CDR_IQD_Pr). Kappa index for the maps interpolated by inverse of the square distance (kappa_IQD_Pr) in function of the coefficient of relative deviation for the maps interpolated by inverse of the square distance (CDR_IQD_Pr).

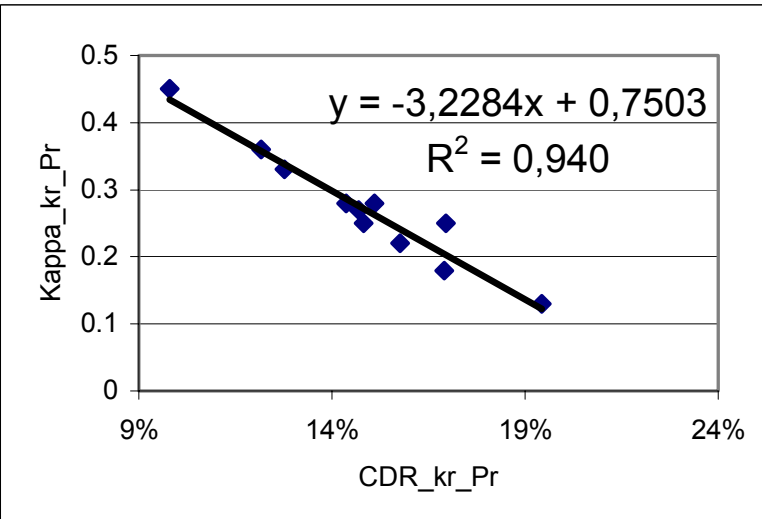
interpolados por krigagem (kappa Kr Pr), em função do coeficiente de desvio relativo para os mapas interpolados por krigagem (CDR_Kr_Pr). Kappa index for the maps interpolated by kriging (kappa_IQD_Pr) in function of the coefficient of relative deviation for the maps interpolated by kriging (CDR_IQD_Pr).

A escolha do número de passadas que podem ser eliminadas do conjunto inicial depende da precisão com que se deseja trabalhar (Tabela 2). Caso se opte pelos níveis arbitrários de 5 e $10 \%$, os números de passadas que podem ser eliminadas, são de 'nenhuma passada' e 'três passadas' no método de interpolação do inverso da distância ao quadrado, e 'nenhuma passada' e 'uma passada' no método krigagem.

TABELA 2. Número de passadas que pode ser eliminado em função da precisão desejada estimada pelo CDR. Step number that can be eliminated in function of the estimated wanted precision by the coefficient of relative deviation.

\begin{tabular}{ccc}
\hline \multirow{2}{*}{ CDR } & \multicolumn{2}{c}{ Produtividade } \\
\cline { 2 - 3 } & IQD & krigagem \\
\hline $3 \%$ & Nenhuma passada & Nenhuma passada \\
$4 \%$ & Nenhuma passada & Nenhuma passada \\
$5 \%$ & Nenhuma passada & Nenhuma passada \\
$7 \%$ & 1 Passada & Nenhuma passada \\
$10 \%$ & 3 passadas & 1 passada \\
$15 \%$ & 10 passadas & 5 passadas \\
$20 \%$ & 25 passadas & 25 passadas \\
\hline
\end{tabular}

$\mathrm{IQD}=$ inverso da distância ao quadrado

\section{CONCLUSÕES}

Nas condições estudadas, a eliminação de passadas da colhedora causou diminuição gradativa na precisão dos mapas de produtividade. 
As interpolações foram influenciadas por ambos os métodos de interpolação (krigagem e inverso da distância ao quadrado), sendo maior a influência do número de passadas eliminadas para os mapas interpolados pelo método de krigagem.

A quantidade de monitores que se deve ter em um conjunto de colhedoras depende da precisão que se deseja, devendo-se intercalar as máquinas de modo que as amostras sejam coletadas uniformemente em toda a área trabalhada.

Para o nível arbitrário de $10 \%$, o número de passadas que podem ser eliminadas, são de 'três passadas', quando se utiliza o método de interpolação do inverso da distância ao quadrado, e 'uma passada' para o método krigagem.

\section{REFERÊNCIAS}

BALASTREIRE, L.A. Estudo de caso, uma pesquisa brasileira em agricultura de precisão, In:

SILVA, FM.; BORGES, P.H. de M. Mecanização e agricultura de precisão. Lavras: UFLA/SBEA, 1998. p.203-32.

COELHO, E.C. Influência da densidade amostral e do tipo de interpolador na elaboração de mapas temáticos. 2006. 116 f. Dissertação (Mestrado em Engenharia de Sistemas Agroindustriais) Universidade Estadual do Oeste do Paraná, Cascavel, 2006.

COHEN J. A coefficient of agreement for nominal scales. Educational and Psychological Measurement, Durham, v.20, p.37-46, 1960.

CONGALTON, R.G. A review of assessing the accuracy of classification of remotely sensed data. Remote Sensing of Environment, St. Paul, v.37, n.1, p.35-46, 1991.

CRESSIE, N.; HAWKINS, M. Robust estimation of the variogram: I. Mathematical Geology, New York, v.12, n 2, p.115-25, 1980.

GOMES, F. P. Curso de estatística experimental. 14.ed. Piracicaba: USP/ESALQ, 2000. 477 p.

LANDIS, J.; KOCH, G. The measurement of observer agreement for categorical data. Biometrics, Washington, v.33, p.159-74, 1977.

MICHELAN, R. Determinação e remoção de erros em mapas de colheita de milho. 2005. $64 \mathrm{f}$. Dissertação (Mestrado em Engenharia de Sistemas Agroindustriais) - Universidade Estadual do Oeste do Paraná, Cascavel, 2005.

SCHUELLER, J.K. O estado-da-arte da agricultura de precisão nos Estados Unidos. In: BALASTREIRE, L.A. (Ed.). O estado-da-arte da agricultura de precisão no Brasil. Piracicaba, 2000. p.8-15.

SHIRATSUCH, L.S.; MACHADO, A.L.T.; FACCIONI, G.C. Geração de mapas de produtividade de milho: efeito do espaçamento entre faixas de colheita. In: CONGRESSO BRASILEIRO DE AGRICULTURA DE PRECISÃO, 2004, Piracicaba. Anais... Piracicaba: ESALQ/USP, 2004. 1 CD-ROM.

TAYLOR, R.K.; KASTENS, D.L.; KASTENS, T.L. Creating yield maps from yield monitor data using multi-purpose grid mapping (MPGM). In: INTERNATIONAL CONFERENCE ON PRECISION AGRICULTURE, 5., 2000, Madison. Proceedings... Madison: ASA/CSSA/SSSA. 1 CD-ROM.

VETTORAZZI, C.A.; FERRAZ, S.F.B. Silvicultura de precisão: uma nova perspectiva para o gerenciamento de atividades florestais. In: BORÉM, A.; GIUDICE, M.P.; QUEIRÓZ, D.M. de. (Ed.). Agricultura de precisão. Viçosa, 2000. p.65-75. 